catarrh with obvious local signs. (2) A generalized " influenzal infection" accompanied by pyrexia.

The diagnosis of a respiratory infection was therefore based on objective evidence which, so far as possible, left no doubt as to the nature of the condition. The diagnosis was not made unless there was sufficient reason for supposing that symptoms and signs were due to recent infection. On this ground the following were excluded: (1) Men with chronic bronchitis. (2) Men with cough but without nasal discharge or pyrexia. Such coughs were attributable to excessive smoking, wax in the ears, training exposures to D.M. gas, or various minor causes. (3) Men who developed coryza or pharyngitis following vaccination: this group presented the only real difficulty, since a considerable but approximately equal number of men in the two units showed inflamed throats or a mild degree of coryza at the height of the vaccine reaction.

The proximity of the two units made it possible, if real doubt arose, for both medical officers to examine the man before a final diagnosis was made. In general the matter was not in doubt, since the great majority of respiratory infections were straightforward examples of acute coryza, pharyngitis, laryngitis, tracheitis, acute bronchitis, or influenza. Over the period of observation both units were receiving recruits at intervals of about a fortnight, so that during the 17 weeks of the experiment a large number of men came under review who were experiencing communal barrack life for the first time.

\section{Oiling of Floors}

In unit $\mathrm{A}$ the wooden floors of all barrack-rooms, sleepinghuts, offices, and lecture-rooms were treated with spindle oil at regular intervals. The oil was applied in such a way as to leave an imperceptible film on the surface after thorough impregnation of the wood. Oil treatment of the floors of unit A began in Nov., 1942, and four applications were made at about monthly intervals up to the end of March, 1943. The oil was non-inflammable and caused no unpleasant smell. The best results were obtained if the floors were first thoroughly swept with a stiff broom, then dry-scrubbed, and finally swept with a soft broom before the oil was applied. This was done with old sacking, which was dipped in the oil, squeezed to get rid of excess, and then well rubbed into the floor along the grain of the wood; no excess of oil was allowed to collect or remain on the floor. The surface dried in about six hours, and one gallon of oil was enough to treat about 1,000 square feet of floor space. Second and subsequent applications made at intervals of about four weeks occupied less time than the first ; care was again taken to ensure that the oil was thoroughly rubbed in and no excess left on the surface. After treatment the floor was not scrubbed, but was easily kept clean by brushing or occasional mopping with a rag moistened in oil. Within the unit the regimental officers and men welcomed the oiling of floors - a point of some importance. The floors were easier to keep clean, scrubbing being unnecessary, and the presence of dirt on the oiled floors was so readily apparent that the men were stimulated to adopt cleanly habits.

The floors of unit B-the control unit-were left untreated, and in both units careful weekly records were kept of all men reporting sick with a respiratory infection during the 17 weeks ending March 27, 1943.

\section{Results}

The results are shown in the accompanying Table. It is clear that the weekly rate of new cases of respiratory infection per 1,000 men was consistently lower in unit $\mathrm{A}$, where the floors were oiled, than in the control unit $B$, where no such treatment of floors was adopted. It is also of importance to note that unit $A$ - suffered no major epidemic of respiratory infection, while unit B sustained a severe outbreak of almost epidemic proportions between the middle of February and the first week of March.

\section{Discussion}

It is not claimed that this is in every respect a perfectly controlled experiment, but the basis of comparison between the two units is probably uniform enough to support the validity of the results. At each unit almost identical conditions prevailed as regards buildings, sleeping-space per man, stress of training, contact with sources of infection outside the barracks, and thas standegd of black-out ventilation. The units were large (streñth 1,300 to 1,700 ) and yielded obvious respiratory infec-
Table showing Weekly Rate of Respiratory Infections per 1,000 Men in Test and Control Units

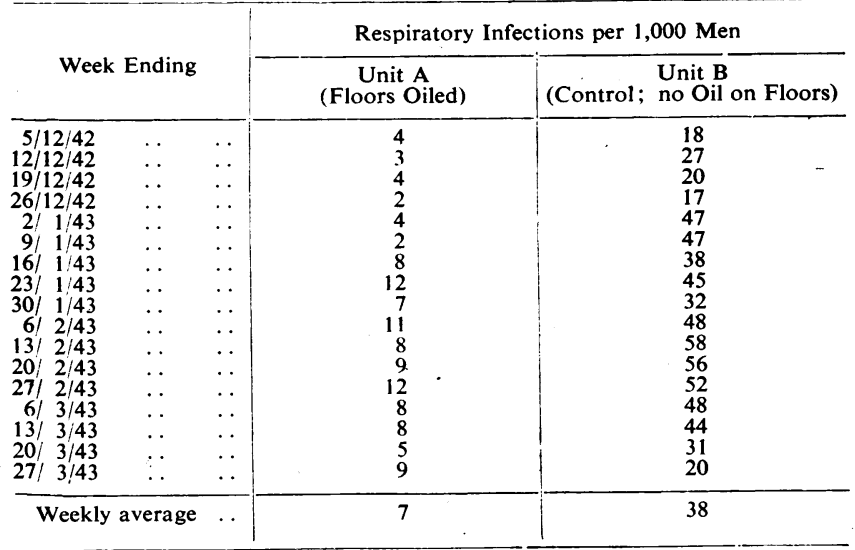

tions in sufficient numbers to reduce the doubtful cases to an insignificant proportion of the whole. Another factor of importance was that close collaboration between the medical officers of the two units helped to eliminate extraneous factors which might have introduced error.

The success in curtailing the spread of purely respiratory infections by oil treatment of floors assumes all the greater significance since it is possible that such important diseases of the barrack-room as virus influenza and cerebrospinal meningitis may also be lessened in their incidence by the same simple procedure.

\section{Summary and Conclusions}

Treatment of wooden floors with spindle oil at regular intervals significantly lowered the rate of respiratory infections in a large military unit as compared with the incidence in a similar control unit, living under comparable conditions, where oiling of floors was not practised.

In the unit where floors were oiled the average rate of respiratory infections was 7 per $1,000 \mathrm{men}$, as against $38^{\circ}$ per $1,000 \mathrm{men}$ in the control unit. During the 17 weeks of the experiment, from the week ending Dec. 5, 1942, to that ending March 27, 1943, no major outbreak of respiratory infection appeared in the test unit; in the control unit an outbreak of almost epidemic proportions prevailed between the middle of February and the first week of March.

\section{REFERENCES}

Allison, V. D., and Brown, W. A. (1937). J. Hyg., Camb., 37, 153. British Medical Journal (1943). 2, 717 (leading article). Cruickshank, R. (1935). J. Path. Bact., 41, 367. (1941). Lancet, 1, 493.

M.R.C. War Memo.' (1941). No. 6, London.

Thomas, J. C. (1941). Lancet, 2, 123.

van den Ende, M., Lush, D., and Edward, D. G. ff. (1940). Ibid., 2, 133. White, E. (1936). Ibid., 1, 941 .

\section{THE DETERMINATION OF BASAL METABOLISM ON OUT-PATIENTS BY}

J. Douglas Robertson, M.D., D.Sc., Ph.D., D.P.H. (From the Courtauld Institute of Biochemistry, Middlesex Hospital)

Measurement of the basal metabolism is not often employed as a routine investigation in this country. One cause may be that most clinics insist that the subjects live in hospital for a few days in order that the test can be carried out at or near the bedside. Such a preliminary preparation is neither popular with the patient nor looked on favourably by the hospital whose beds are already outnumbered by patients requiring urgent medical and surgical treatment. In this paper proof will be given that in fact it is not necessary to use hospital beds for this test and that determinations on ambulatory patients are satisfactory. At the same time certain technical points on the measurement, accuracy, and constancy of the basal metabolism will have attention drawn to them.

The method of determining the metabolism was that of the closed circuit, the accuracy of which has already been reported (Robertson, 1937). The apparatus used was the Benedict-Roth with recording kymograph. All machines were alcohol-checked 
(Barrett and Robertson, 1937), but in addition each is checked once a week by a model whose basal metabolism is in the region of 56 calories an hour.

\section{Findings}

In the tables which follow, an attempt is made to answer some of the controversial problems in metabolism, such as the number of observations necessary to obtain an accurate result, the effect of exercise on the basal metabolism, and the accuracy of determinations on ambulatory patients.

First, 100 patients were studied on two consecutive days, with duplicate readings each of 10 minutes a day; the results are given in Table I. No significant difference was found

TABle I.-A Study of 100 Consecutive Patients having Two Tests on Two Consecutive Days

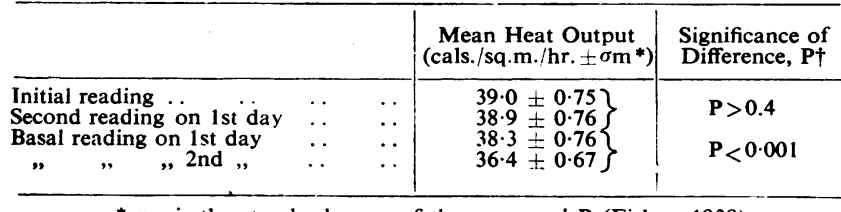

* $\sigma \mathrm{m}$ is the standard error of the mean. + $\mathrm{P}$ (Fisher, 1938).

between the two readings taken on the first day $(t=0.895$; $P>0.4$ ). There was, however, a significant fall in the basal metabolism on the second day compared with the first $(t=8.3$; $\mathbf{P}<0.001$ ). This finding in addition to a smaller standard deviation on the second day favours a study of the patient on at least two days.

Next, in Table II, are a series of 26 subjects studied on two successive days as before; but on the afternoon previous to the first standard reading two practice tests, of the same

TABLE II.-A Study of 26 Subjects having Two Tests on Two Consecutive Days (but Two Practice Tests similar in Duration to the Basal Tests were carried out on a Previous Afternoon)

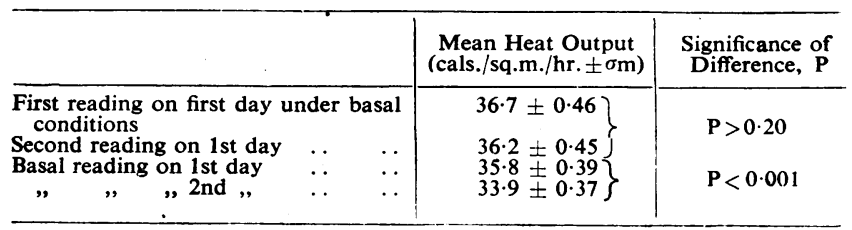

duration as the actual tests, were carried out. The element of training was thus introduced before the first observation under basal conditions. Despite this, however, a significant fall in the metabolism took place on the second day $(t=6.4$; $P<0.001)$. Once again the two first-day readings were not significantly different $(\mathrm{t}=1.34 ; \mathrm{P}>0.20)$. Thus whereas $\mathrm{a}$ dummy reading or practice test is often of value in relieving the anxiety of some patients, it cannot replace the test, under basal conditions, carried out on two separate days.

To determine whether the metabolism continued to fall as the number of observations increased, a series of 14 subjects were studied on six successive days; the results are given in Table III. Again a significant fall on the second day was

TABLE III.-A Study of 14 Subjects having Two Tests on Six Consecutive Days

\begin{tabular}{|c|c|c|c|}
\hline & & $\begin{array}{l}\text { Mean Heat Output } \\
\text { (cals./sq.m./hr. } \pm \sigma \mathrm{m})\end{array}$ & $\begin{array}{l}\text { Significance of } \\
\text { Difference, } P\end{array}$ \\
\hline 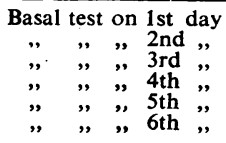 & $\begin{array}{ll}\because . & . \\
\cdots & \cdots \\
\because . & \cdots \\
\cdots & \cdots \\
\cdots & \cdots\end{array}$ & $\left.\begin{array}{l}35.5 \pm 0.96 \\
33.5 \pm 0.75 \leftarrow \\
33.5 \pm 0.80 \\
33.6 \pm 0.83 \\
33.5 \pm 0.75 \\
33.1 \pm 0.83\end{array}\right\}$ & $\mathrm{P}<0.001$ \\
\hline
\end{tabular}

noticed $(t=4.07 ; P<0.001)$. But a comparison of the second day with the sixth day (the days with the maximum disparity, excluding the first-day tests) showed no significant difference $(t=1.36 ; P>0.2)$. Thus by the second day a reproducible basal metabolism can be recorded.

The subjects studied above in Table III were normal healthy volunteers (nurses). A similar study was now made on persons suffering from thyrotoxicosis, and these are giveh' in 'Table IV.
TABLE IV.-A Study of 7 Cases of Thyrotoxicosis having Two Tests on Seven Consecutive Days

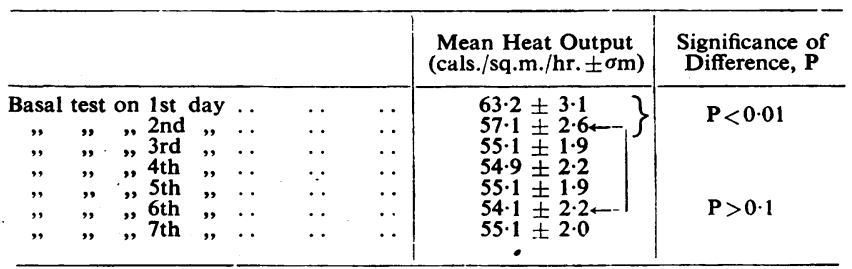

As with normal subjects, a significant fall in metabolism occurred on the second day $(t=3.51 ; P<0.01)$. The fall continued to take place daily, reaching its maximum on the sixth day, but a statistical study showed that this further fall is of no significance. If the second- and sixth-day readings are compared (the days with the maximum disparity in their means) no significant difference is found $(t=1.73 ; P=0.1)$. Thus cases of thyrotoxicosis by the second day give a satisfactory and reproducible reading of their basal metabolism.

In Table $\mathrm{V}$ the effect of exercise on the basal metabolism is shown. The subjects (12 normal, and 5 cases of thyrotoxicosis) were first studied under basal conditions. After this

TABLE V.-A Comparison of the Metabolism under Basal Conditions
with the Same Metabolism following Exercise. After the
exercise the subjects lay down on a couch and the
metabolism was measured without preliminary
rest for the next 40 minutes

reading was taken, they rose, and descended and ascended the steps of the Courtauld Institute (95 steps; distance down, 55 feet) in approximately $2 \frac{1}{4}$ minutes. In both normal and thyrotoxic subjects the metabolism again became basal after a rest of only 20 minutes.

Finally, a comparison was made between the metabolism measured under strictly basal conditions and that under ambulatory conditions. Full details of the routine carried out by four subjects after rising and leaving their homes for the metabolism department are given in Table VI. Despite in some cases quite appreciable exercise and the exposure to an outside temperature about freezing-point, half an hour's repose on a couch in a room at a temperature of 20 to $23^{\circ} \mathrm{C}$. was adequate to reproduce basal conditions.

\section{Discussion}

Different clinics do not appear to have the same system of assessing a subject's basal metabolism. There is no unanimity about the number of tests, the number of daily observations, and whether to report a mean of all results or the lowest reading. In some clinics it is the usual custom to carry out only a single determination without any preliminary practice, thus excluding the element of training (Boothby, Berkson, Dunn, 1936). Those authors, however, add that the initial reading is accepted "unless at the time of the test and before its calculation it was noted as unsatisfactory for reasons of restlessness, observable nervous tension, or an elevated temperature.". There are several objections to Boothby et al.'s technique. First, so-called nervous tension is not uncommon at the initial test, and particularly in cases of thyrotoxicosis. Secondly, initial readings are commonly higher, and sometimes much higher, than subsequent or true basal determinations. Thus in a series of 223 subjects aged 19 , $156(70 \%)$ gave initial readings which were significantly higher than those on the second day, $48(22 \%)$ gave results $110 \%$ or more higher, $21(9 \%)$ gave results $15 \%$ more thigher, 
TABlE VI.-A Comparison of the Bedside and Ambulatory Estimations of the Basal Metabolism

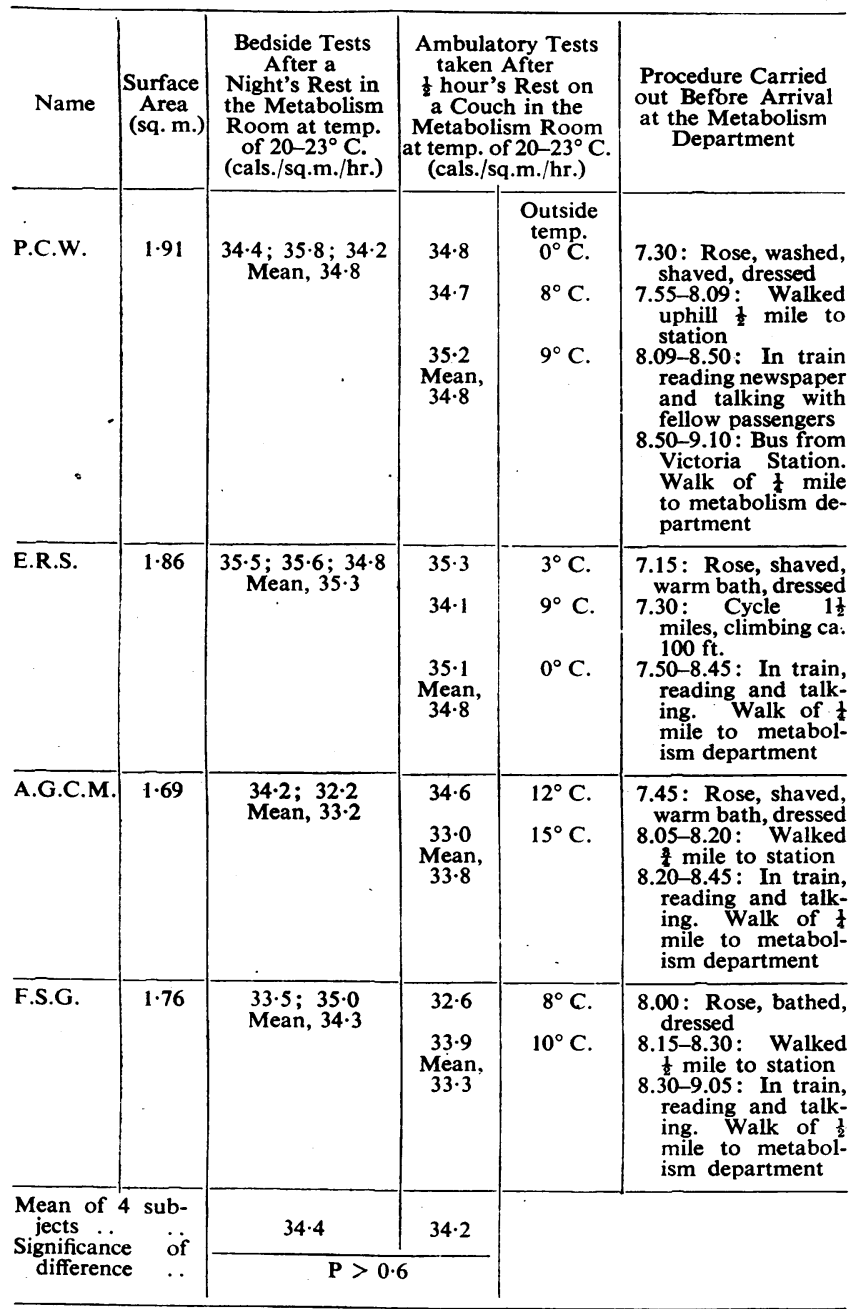

and as many as $11(5 \%)$ gave readings as elevated as $20 \%$ higher. In no case was the initial reading at the time of the test considered unsatisfactory. The series was a group of nurses who had volunteered for the test. To judge a subject's basal metabolism on only a single observation is to run the risk of reporting a raised instead of a normal metabolism. Thirdly, if standards are based on initial readings then the metabolic assessment of subjects who have ever had the test before becomes difficult. The experience of a test previously means training which Boothby et al. suggested should be excluded. Finally, the controlling influence of iodine on the basal metabolism of thyrotoxicosis as a diagnostic test becomes much less sensitive; for, indeed, it would be very difficult to determine whether a fall in the metabolism in a subsequent test after iodine was due to the element of training or to iodine.

In other clinics several readings are taken on one day, and either the mean or the lowest reading is accepted as the basal metabolism. Other clinics prefer observations carried out on several days, and again the mean or the lowest reading is taken as the basal metabolism. As the technique for assessing a subject's basal metabolism appears to vary in different clinics, one must expect some disparity in their respective results.

The findings in this paper are that by the second day of basal conditions, with few exceptions, a reproducible result can be obtained for the basal metabolism of normal people and cases of thyrotoxicosis that is constant over: a period of several days at least. It has been the custom in this department, therefore, to adopt a certain technique in measuring and reporting on the basal metabolism: it is the attendance of the subject on two successive mornings after the customary 'ast of least 12 hours. After half an hour's rest on a couch in a room at a temperature of 20 to $23^{\circ} \mathrm{C}$. duplicate determinations, each of 10 minutes' duration, are taken. On the second day the results are commonly lower than on the first; but if this is not so, the subject is asked to attend on another morning. On the day with the lowest reading the duplicates are expected to agree within $5 \%$ (or attendance on a further day is requested), and the lower of the two readings is reported as the basal metabolism.

It is suggested that the measurement of the basal metabolism in this country is not commonly carried out because insufficient use is made of ambulatory determinations. There is a reluctance to accept results on ambulatory patients because they are believed to be inaccurate. This is an entirely false conception without any experimental evidence to support it. Proof has here been shown that in fact metabolism determinations on ambulatory subjects are as accurate, uniform, and reliable as those carried out at the actual bedside if half an hour's repose on a couch in a warm room precedes the determination. Benedict and $\mathrm{Du}$ Bois, in a personal communication, stated this interesting fact many years ago, and their findings were confirmed at that time. It is interesting to note that cases of thyrotoxicosis as well as normal subjects, in so short a period as 20 minutes' rest on a couch, can reproduce basal conditions even after such exercise as walking down 95 steps and up again in $2 \frac{1}{4}$ minutes. From a diagnostic point of view it is unfortunate that cases of thyrotoxicosis respond no differently to exercise than do normal subjects. For if, in thyrotoxic cases; half an hour's repose on a couch was insufficient to reproduce basal conditions, the effect of exercise on the metabolism might constitute a sensitive diagnostic test for mild cases of thyrotoxicosis. Attention has been drawn to these mild cases of thyrotoxicosis with a basal metabolism apparently normal yet actually raised (Robertson. 1934). Iodine in conjunction with the basal metabolism will invariably detect these mild cases. In that paper a proved case of thyrotoxicosis was described in which an initial basal metabolism of minus 8 fell to minus 27 on iodine (i.e., minus 27 was normal for the patient, so minus 8 meant a raised basal metabolism)

It is believed that the value of ambulatory metabolism determinations is still insufficiently appreciated in this country. The blocking of a hospital bed for several days for an examination of this nature adds considerably to its cost, and the patient is unwilling to co-operate because of loss of working hours, personal expense, and the like. The results given above indicate that there is no reason why an out-patient should not attend hospital in the morning for the test (approximate time, 1 hour), then return to his ordinary duties for the rest of the day. A test carried out early enough might result in no loss of working hours.

\section{Summary and Conclusions}

A reproducible metabolism measurement can be obtained if the subject attends for the test on at least two mornings under basal conditions. By the second day of the test, if the duplicates agree within $5 \%$, then the lower of the readings can be taken as that subject's basal metabolism. If the second-day tests are higher than those taken on the first day, then further attendance is advisable until a constant reading is obtained.

The above technique can be applied with satisfaction to normal subjects and cases of thyrotoxicosis.

After reasonable exercise basal conditions can be reproduced after resting on a couch for approximately 20 minutes. A preliminary repose of half an hour before metabolism reading is taken is adequate for basal purposes.

A satisfactory, accurate, and reproducible metabolic reading can be obtained on " out-patients." These so-called " ambulatory determinations" are not significantly different from those tests carried out at the bedside under strictly basal conditions.

The reproducibility of the method described above for measuring the basal metabolism is within $5 \%$.

It is a pleasure to thank Prof. E. C. Dodds for his criticism of this paper and the Government grants for scientific investigation, through the Royal Society, for the gift of a basal metabolism machine.

\section{REFERENCES}

Barrett, J. F., and Robertson, J. D. (1937), J. Path. Bact., 45, 555. Boothby, W. M., Berkson, J., and Dunn, H. L. (1936). Amer. J. Physiol., 116, 468

Statistical Methods for Research Workers, 7th ed., p. 128. Edinburgh, Oliver and Boyd.

Robertisón, J ț. (1934)) Lancet, 1934, 2!14076.

$4(1937)$. Lhid., 2, 815.

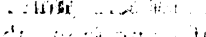

